\title{
SDN Control Architectures for WDM over SDM (WDMoSDM) Networks
}

\author{
R. Muñoz ${ }^{(1)}$, N. Yoshikane ${ }^{(2)}$, C. Manso ${ }^{(1)}$, R. Casellas $^{(1)}$, R. Vilalta ${ }^{(1)}$, \\ R. Martínez ${ }^{(1)}$, F. Balasis ${ }^{(2)}$, C. Wang ${ }^{(2)}$, T. Tsuritani ${ }^{(2)}$, and I. Morita ${ }^{(2)}$ \\ ${ }^{(1)}$ Centre Tecnològic de Telecomunicacions de Catalunya (CTTC/CERCA), Castelldefels (Barcelona), Spain \\ ${ }^{(2)}$ KDDI Research, Inc., Saitama, Japan \\ Corresponding author:raul.munoz@cttc.es
}

\begin{abstract}
We provide an overview of SDN control architectures for WDM over SDM (WDMoSDM) optical networks. We address dynamic management of end-to-end connectivity services and WDM virtual network topologies (VNTs) for partially disaggregated multi-domain WDMoSDM networks. (C) 2021 The Author(s)
\end{abstract}

\section{Introduction}

Telco operators are facing two main challenges in the deployment and operation of their transport networks; cost reduction and capacity increase. The first challenge is addressed by targeting the partial disaggregation of the optical networks. In a first stage, the line system (i.e., ROADMs, optical amplifiers, etc.), is open to transponders from different vendors. It enables telco operators not to be bounded to a single vendor for new transponder deployments, but still enabling a single vendor to have full responsibility of the open line system (OLS). The second challenge is addressed by deploying Spatial Division Multiplexing (SDM). A novel network architecture that has been recently proposed to increase the capacity of the optical transport systems is spatial channel network (SCN) [1]. SCN allows to bypass the overloaded wavelength division multiplexing (WDM) networks by provisioning spatial paths between WDM nodes. It is composed of spatial cross connects (SXC) that enable to provision spatial channels that occupy the entire available spectrum of a single-mode fiber (SMF) or one core in a multi-core fiber (MCF).

In this paper we provide an overview of first presented SDN control architectures for dynamic management of end-to-end connectivity services and WDN virtual network topologies (VNTs) for partially disaggregated multidomain WDM over SDM (WDMoSDM) networks.

\section{Dynamic management of end-to-end connectivity services}

In [2] and [3], we presented and experimentally validated the first TAPI-enabled SDN control architecture for partially disaggregated multi-domain (OLS) and multi-layer (WDM over SDM) optical networks. Fig.1.a shows an example of the target network. It is composed of multiple OLS domains and transponders leveraging WDM and SDM technologies. WDM OLS domains are deployed using WDM nodes (e.g., ROADMs) that provide optical channels. SDM OLS domains provides spatial channels between WDM nodes or between SDM transponders. The considered SDN control system architecture relies on an optical SDN controller that orchestrates the OLS controllers and the transceivers based on open APIs. The interface considered for the OLS controllers is based on Transport API (TAPI). TAPI defines a common YANG data model for control services such as context retrieval, connectivity management, and path computation services.

A TAPI client can request a TAPI digital signal rate (DSR) connectivity service between two transponders specifying a digital rate (e.g, 100 Gigabit Ethernet). First, the SDN orchestrator computes an end-to-end path to serve the request and select the involved OLS domains. If an SDM OLS domain is required, the SDN controller requests a spatial channel to the SDM OLS controller and augments the internal topology with a new inter-domain link between the WDM nodes associated to the provisioned spatial channel. Once the spatial path is provisioned, the SDN orchestrator computes the spectrum required (in $\mathrm{GHz}$ ) to serve the requested capacity, and requests to each involved WDM OLS controller the computation of the intra-domain path. We have also extended the TAPI path computation service in order to provide the available spectrum along the computed path. It is used to compute a common frequency slot that meets the spectrum needs and is available across all WDM domains. After that, the SDN orchestrator requests the provisioning of the optical channel on each WDM OLS domain through the TAPI connectivity service, specifying the selected frequency slot. The last step performed by the Transport SDN orchestrator is to configure the transponders. The SDN orchestrator computes the required modulation to meet the requested capacity for the provisioned optical channel and configures the transmitter and the receiver using an open API. 


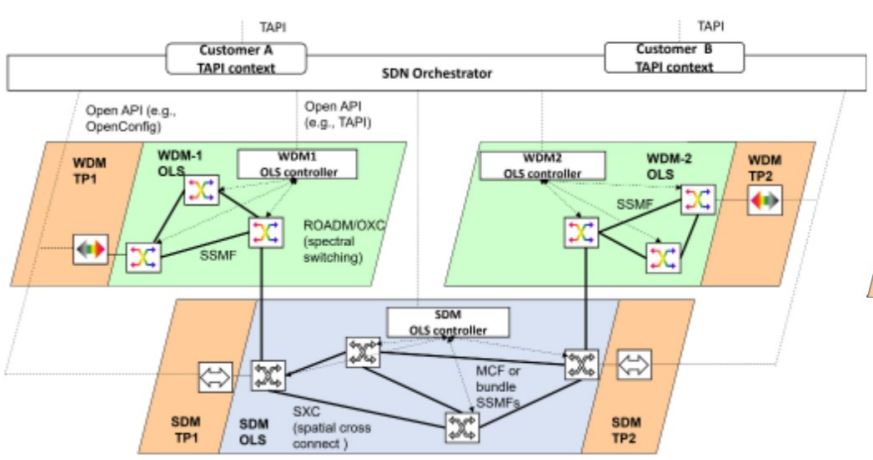

(a) End-to-end connectivity services

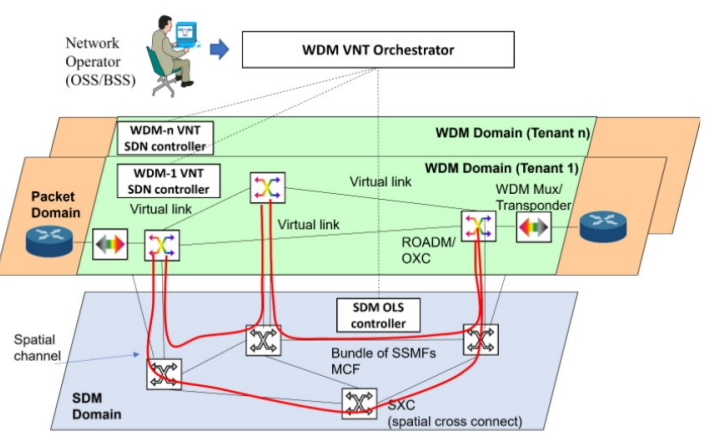

(b) WDM VNT services

Fig. 1: SDN control architectures for partially disaggregated multi-domain WDMoSDM networks

\section{Dynamic management of WDN virtual network topologies}

In [4], we took advantage of the WDMoSDM networks to extend the concept of virtual network topologies (VNTs) to the WDM layer. In IP over WDM networks, VNTs forming logical IP networks are created by connecting real IP routers through virtual links provided by lightpahts in the WDM layer. Similarly, we propose the dynamic deployment of WDM VNTs. It consists on providing logical WDM networks by deploying virtual links between real WDM nodes using spatial channels provided by the SDM layer. It supports multi-tenancy, as a single SDM network can provide virtual WDM links for different WDM VNTs. The spatial channels can be dynamically allocated to the WDM VNTs based on some constraints, such as link disjointness (e.g, to avoid virtual links from a WDM VNT use the same MCF) or reduced inter-core crosstalk. We proposed a cloud-native SDN controller based on micro-services acting as WDM VNT orchestrator for the lifecycle management of the WDM VNTs. The cloud-native SDN controller is based on the $\mu \mathrm{ABNO}$ [5], an SDN controller with a microservices architecture.

The network operator is responsible for defining the logical WDM VNT (i.e., involved WDM nodes and required virtual links). Once defined, it requests the provisioning of the WDM VNT to the WDM VNT orchestrator. First, the WDM VNT orchestrator gets the TAPI context of the WDM and SDM domains. The TAPI context includes the topology and the active connectivity services (i.e., optical and spatial connections). Then, the WDM VNT orchestrator computes the spatial channel for each virtual link included in the requested WDM VNT using a VNT assignment algorithm. After a suitable spatial channel is found for each virtual link, the WDM VNT orchestrator requests the provisioning of the SDM TAPI connectivity services to the SDM OLS Controller, specifying the computed spatial channel. After that, the WDM VNT Orchestrator requests the WDM VNT SDN controller to update its topology with the newly created virtual links. This way, the WDM nodes are connected by virtual links deployed as spatial channels that are transparent to the WDM domain. Finally, the WDM VNT orchestrator gets the WDM and SDM domain TAPI contexts to check all operations are performed correctly before it replies.

\section{Conclusions}

This paper has reviewed the first SDN control architectures for end-to-end connectivity and WDM VNT management in partially disaggregated multi-domain WDMoSDM networks.

\section{Acknowledgments}

Work supported by the Spanish project AURORAS (RTI2018-099178-B-I00), EC H2020 TERAFLOW project (101015857), and the NICT Japanese project "Massive Parallel and Sliced Optical Network".

\section{References}

1. M. Jinno, "Spatial channel network (scn): Opportunities and challenges of introducing spatial bypass toward the massive sdm era," J. Opt. Commun. Netw. 11, 1-14 (2019).

2. R. Muñoz, N. Yoshikane, R. Vilalta, R. Casellas, R. Martínez, T. Tsuritani, and I. Morita, "Network control and orchestration in sdm and wdm optical networks," in Optical Fiber Communication Conference, (Optical Society of America, 2020), pp. T3J-2.

3. C. Manso, R. Muñoz, N. Yoshikane, R. Casellas, R. Vilalta, R. Martínez, T. Tsuritani, and I. Morita, "Tapi-enabled sdn control for partially disaggregated multi-domain (ols) and multi-layer (wdm over sdm) optical networks," J. Opt. Commun. Netw. 13, A21-A33 (2021).

4. C. Manso, R. Muñoz, F. Balasis, R. Casellas, R. Vilalta, R Martinez, N. Yoshikane, C. Wang, N. Yosikane, T. Tsuritani, and I. Morita, "Tapi-enabled sdn control for partially disaggregated multi-domain (ols) and multi-layer (wdm over sdm) optical networks," in European Conference on Optical Communication (ECOC), (2021).

5. C. Manso, R. Vilalta, R. Casellas, R. Martínez, and R. Muñoz, "Cloud-native sdn controller based on micro-services for transport networks," in 2020 6th IEEE Conference on Network Softwarization (NetSoft), (IEEE, 2020), pp. 365-367. 